\title{
Decomposition analysis and peak stagger design for Crank-triangular linkage-elbow mechanism of mechanical servo presses
}

\author{
Yousong Sun ${ }^{1, *}$, Jianguo $\mathrm{Hu}^{2}$, Liangmo $\mathrm{Wei}^{1}$, and Yongqi Chen ${ }^{1}$ \\ ${ }^{1}$ School of Material and Energy Engineering, Guangdong University of Technology, Guangzhou 510006, PR China \\ ${ }^{2}$ Mechatronic Engineering Department, Shunde Polytechnic Institute, E. Desheng Rd., Shunde 528333, PR China
}

Received: 15 February 2020 / Accepted: 25 August 2021

\begin{abstract}
High mechanical advantage as well as low and steady slide speed within the working stroke $S_{n}$ are the fundamental requirements for the working mechanism of servo-mechanical press. Currently, the CrankTriangular Linkage-Elbow (CTLE) mechanism has attracted more and more attention from researchers and manufacturers of servo presses. This paper presents a new analysis and design method of CTLE. The mechanism is decomposed into two sub-units: crank and triangular-linkage elbow, followed by the kinematic and force analysis of each sub-unit. The influences of each structural parameter on the working performance are obtained and can be used as the basis for preliminary design. Through the offset design, the mechanical advantage peaks of the two units, $M A_{1 \max }$ and $M A_{2 \max }$, do not occur at the same time: $M A_{1 \max }$ is located near $S_{n}$, while $M A_{2 \max }$ is just at BDC (Bottom Dead Center). Because the mechanical advantage of the whole mechanism is the product of the two subunits, the designed mechanism can obtain high and steady mechanical advantage together with low and steady slide speed within $S_{n}$. After preliminary design, the scheme can be further modified by numerical simulation and optimization. Hence the design efficiency can be improved.
\end{abstract}

Keywords: optimal design / mechanical advantage / mechanism / multi-linkage / mechanical servo press

\section{Introduction}

Mechanical press is the most popular forging and stamping equipment applied in industry, and its driving system includes power source (motor), reducing system and working mechanism, whose function is to convert the input rotary motion into linear reciprocating motion of the slide [1].

The driving system of the traditional mechanical press has a flywheel and its design is based on energy. The basic design principles are: (1) The whole slide stroke is divided into idle stroke and working stroke in which the workpiece is deformed. (2) The nominal pressure stroke $S_{n}$, an important performance parameter for the mechanical press, is defined as a specific slide position above BDC, while the corresponding crank angle is called the nominal pressure angle $\alpha_{n}$. $S_{n}$ is considered as the working stroke in the design. (3) The nominal pressure $P_{n}$, another important parameter, is the maximum allowable load for the press within $S_{n}$. When the slide is outside the range of $S_{n}$, the allowable load will be less than $P_{n}$. (4) The key

\footnotetext{
* e-mail: ys19440139.com
}

consideration of the driving system design is the flywheel, and the design criterion is that the released energy from the flywheel deceleration within a certain range (e.g., 20\%) should be equal to the energy consumed in a working cycle [2].

The servo mechanical press emerged at the end of the last century is a new type of forging and stamping equipment. The common inductive motor is replaced by the servo motor and the flywheel, clutch and brake are eliminated in the driving system. This innovation not only simplifies the transmission chain and reduces the energy consumption, but also changes the working characteristics of the press from uncontrollable to controllable, improving the flexibility and automation of the equipment [3-5]. Owing to the reforms in the driving system, the design criteria for servo press are quite different. Torque becomes the basis instead of energy: the input torque during the working stroke should be able to overcome the working load during the working stroke.

The price of a servo mechanical press is relatively high, owing to the expensive servo motor with large torque. How to reduce the servo motor capacity has been a hot topic in the current development of the large-tonnage servo mechanical press. 
Table 1. comparison of four mechanisms.

\begin{tabular}{|c|c|c|c|c|}
\hline & Screw & Eccentric & Knuckle & CTLE \\
\hline Sche-matic diag-ram & & & & $\psi$ \\
\hline$V n$ & constant & high & low & lower \\
\hline$V V n$ & constant & large & small & smaller \\
\hline$M A n$ & constant & low & high & higher \\
\hline size & large & small & large & larger \\
\hline
\end{tabular}

Note $S_{\text {max }}$ : Maximum stroke; $\bar{V}_{n}$ : average velocity in $S_{n} ; V V_{n}$ : velocity variation; $M A_{n}: M A$ in $S_{n}$.

One approach is to use hybrid driving power $[6,7]$ with the flywheel in the transmission system retained, which will reduce the press flexibility. Another approach is to adopt multi-motor parallel drive, which has been widely used in large and medium-tonnage servo presses, but it will be more demanding for the control and transmission systems [8].

Selecting the appropriate working mechanism and optimizing the design would be the most cost-effective and efficient way. The ideal working mechanism should have large force amplification factor (high mechanical advantage) and low and steady speed output in the working stroke $[1,3,5]$.

Based on kinematics and dynamics analysis of the working mechanism, the author puts forward the design requirements for the working mechanism of servomechanical press: (1) high and steady mechanical advantage in working stroke $S_{n}$; (2) low and uniform slide speed in $S_{n}$; (3) high slide speed in idle stroke; (4) the stroke of slide being a monotonically increasing function of the crank angle within the range between TDC (Top Dead Center) and BDC [1].

The working mechanisms of the mechanical servo press are similar to those used in the traditional mechanical press, which include four types: screw, eccentric drive (crank-connecting rod, eccentric shaft and eccentric gear, etc.), knuckle-joint and linkage mechanism [4,5]. The linkage mechanism has several names in literatures, such as modified knuckle-joint [2], linkage [4], toggle [5], etc. In order to better characterize its structural features, it is called Crank-Triangular Linkage-Elbow mechanism (CTLE) in this paper. CTLE has attracted extensive attention from researchers and manufacturers of servo presses owing to its good kinematic and dynamic characteristics [4,5,9-12]. Especially, CTLE has a high mechanical advantage and low and steady speed in $S_{n}$, meeting the requirements for mechanical servo press $[1,11]$. Table 1 shows the comparison of the four mechanisms and Figure 1 shows their stroke-time curves.

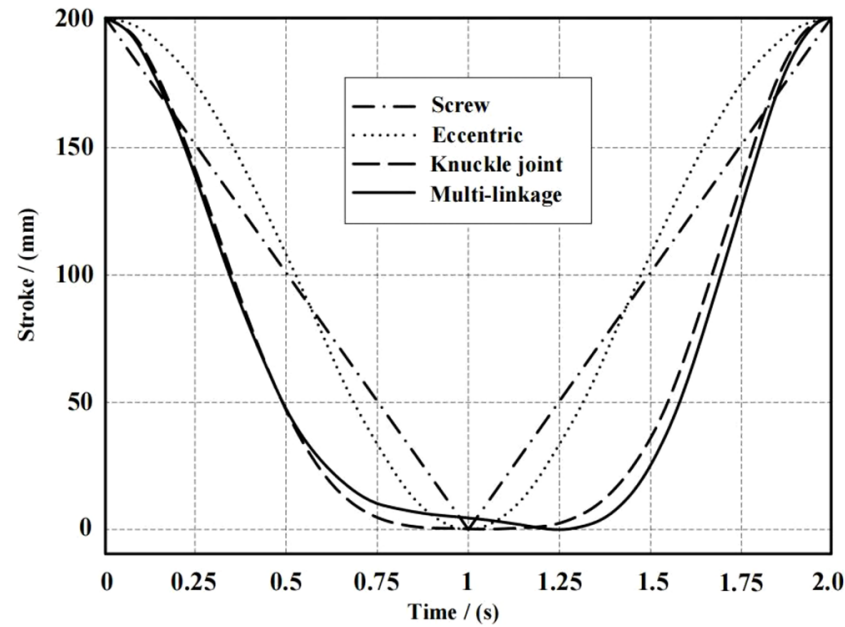

Fig. 1. Stroke-time curves of four mechanisms.

The working mechanism of servo mechanical press is usually the planar linkage mechanism, and its modeling, simulation and optimization have been a hot issue in mechanism research. Analytical method could only solve some relatively simple problems [13]. In recent years, numerical simulation technology provides a powerful tool for solving complex linkage system problems. Halicioglu proposes a modeling and design method for a crank servo press based on Lagrangian [14]. In [15,16], the SimulationX software package was used in dynamic modelling of a servo mechanical press. Dynamic model of the servo mechanical press was composed with account for elastic-dissipative characteristics of the links and the parameters of the servomotor [15]. In [13], Sequential Quadratic Problem (SQP) approach was used to reduce the probability of branch or circuit defects in optimization. In [17], the synthesis and optimization of two types of mechanism are 
conducted by optimization methods of objective function method and performance atlases. Tuleshov et al. considered that the results of the numerical synthesis of the linkages depend on the choice of the initial approximations. In order to find the initial approximations, a method based on the use of Burmester points was proposed [18]. Reference [19] presents a newly developed hybrid simulated annealing-direct search algorithm (SADS) for the optimization of mechanism synthesis. Amandyk Tuleshov et al. present a numerical method for kinematic synthesis of the Stephenson mechanism. It is observed that eccentricity of Stephenson mechanism affects the lower forward and higher backward speed of the slider. The literature only provides numerical analysis of kinematics, lacking dynamic analysis [10].

In summary, the general approach to solve the optimization problem of planar linkage system is: (1) establish the mathematical model of the system through analysis; (2) numerical simulation is conducted by proper software tools. At present, there are many software platforms, such as ADAMS, MATLAB, KATIA, SimulationX and so on. (3) Optimize the system through appropriate approach. André Höber et al. developed a software for servo press transmission system design, but it is only suitable for crank and toggle mechanisms, and can only solve the kinematic simulation, without involving the optimization of the system [20].

Although the working mechanism of servo press is basically the same as that of common mechanical press, their design principles are quite different, due to the different working requirements. For the common mechanical presses, the design of the working mechanisms usually focuses on the kinematic characteristics: proper and steady speed in the working stroke, meeting the requirements of the forming process [21-24]. The mechanical advantage, although mentioned in some documents for mechanism optimization, is usually not a key factor. For example, Hwang listed the mechanical advantage as one of the seven objective functions in the design of a mechanical press, but its weight factor was only 50, while it was up to 1000 for the variance of the slide speed [23]. Most of the research on optimization of the working mechanism for mechanical servo presses considered the mechanical advantage as the main objective.

Yossifon et al. [25,26] analyzed various servo-driven multi-link mechanisms and proposed an optimization scheme with the objectives of maximum mechanical advantage and minimum structural dimensions. Within the defined domain, the mechanical advantage in the working stroke increased from 2.5 to 4.0 , with a slight reduction of structural dimensions. Guo et al. [27] optimized the servo press by performance atlases, taking the reciprocal of mechanical advantage and the ratio of connecting rod load to output force as performance indicators. Du et al. [28] optimized the design by genetic algorithm, using feasibility conditions, mechanical advantage and motor torque as objective functions. Wang Zong Qiang [29] proposed a screw-driven triangular linkagetoggle mechanism for servo mechanical press, and carried out kinematic analysis. The influences of structure parameters on mechanical advantage were studied by the combination of digital simulation and trial-and-error. Jianguo $\mathrm{Hu}$ [11] studied the CTLE mechanism of servo mechanical press and established an optimization method, aiming at maximum mechanical advantage and best kinematical performances.

Methods of mechanism optimization can be divided into three kinds: heuristics, numerical and analytical. The analytical is intended for the analysis of concrete and not very complex mechanisms. The synthesis of mechanisms is a heavily nonlinear problem, and this usually leads to resort to iterative procedures whose convergence may not always be guaranteed [13]. Moreover, the optimization process is not only time-consuming, but also difficult to obtain the global optimal solution because the initial conditions and the constraints of the mechanism are often determined by designer's experiences [17].

By mathematical analysis on kinematics and dynamics of the mechanism, the general rule of the influence of the structural parameters on the performance of the mechanism can be obtained. On this basis, the initial model, constraints and optimal path of the optimal design can be established, which will help to simplify the design process, obtain the optimal solution and improve the design efficiency.

\section{Decomposition analysis method on complex linkage mechanism}

Decomposition analysis is an effective method to solve the modeling problem of the complex planar linkage mechanisms. The most well-known is the so-called Assur group method [30-32]. An Assur group is a neutral module with 0 DOF (Degree of Freedom), mathematically corresponding to equation (1).

$$
3 n-2 p_{l}-p_{h}=0
$$

where $n$ is the number of bodies, $p_{l}$ and $p_{h}$ are the numbers of low and high pairs in the module respectively.

The main purpose of the traditional decomposition analysis is for modelling. The kinematic and dynamic solutions obtained are mostly in the form of matrix or vector, which is very convenient for numerical simulation.

The decomposition method proposed in this paper is slightly different from the traditional approach. Its purpose is to obtain the influence rule of the structural parameters and the combination form of the adjacent subunits on the working performance. The rule can be the guide for the preliminary configuration design of the mechanism. The subunit is either an Assur group or a combination of several Assur groups, which will not be divided further if their connecting form in the subunit is relatively fixed.

As analyzed in Section 1, the mechanical advantage of the working mechanism $M A$ is one of the most important parameters for a servo mechanical press. Figure 2 is the schematic diagram of mechanism decomposition. The subunits are connected in series with hinges and the output of each subunit is the input of the next. The 


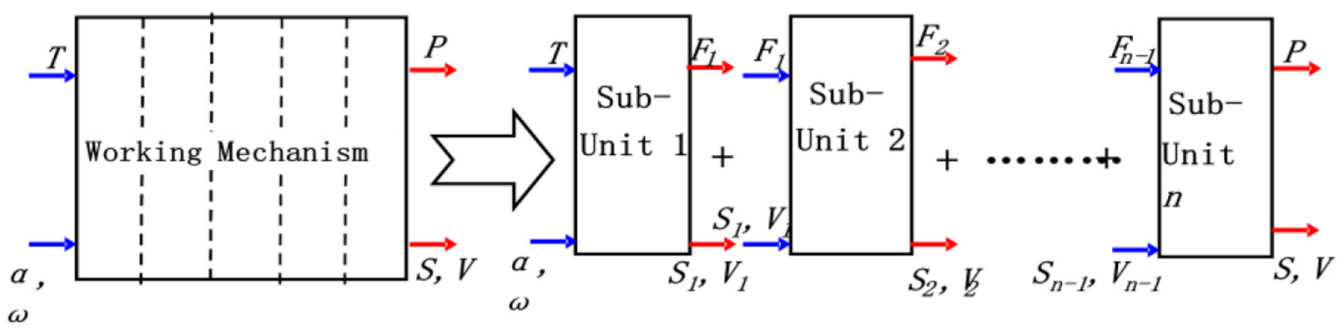

Fig. 2. Schematic diagram of decomposition for complex linkage mechanism.

mechanical advantage $M A$ of the whole mechanism can be expressed as equation (2).

$$
M A=\frac{P}{T}=\frac{F_{1}}{T} \cdot \frac{F_{2}}{F_{1}} \cdots \frac{P}{F_{n-1}}=\prod_{1}^{n} M A_{i}
$$

where $T$ is the input torque and $P$ is the slide output pressure, while $F_{i}$ and $F_{i+1}$ refer to the input and output forces of sub-unit $i+1$ respectively; $S_{i}, V_{i}$ and $S_{i+1}, V_{i+1}$ represent the input displacement, speed and output displacement and speed of the subunit $i+1$ respectively; $M A_{i}$ is the mechanical advantage of subunit $i, i=1 \sim n$.

As for a crank-slide linkage mechanism, the mechanical advantage $M A$ of the mechanism can be expressed as equation (3) [33].

$$
M A=\frac{P}{T}=\frac{\omega}{V}
$$

where $T$ and $\omega$ are the torque and angular velocity input by the crank, and $P$ and $V$ are the force and velocity output by the slide. Due to the nonlinearity of the linkage mechanism, $M A, T, P$ and $V$ are probably variables, even if $\omega$ is constant. When $\omega$ is constant, $M A$ will be inversely proportional to $V$, which means the slide speed will decrease with the increase of the mechanical advantage.

\section{Decomposition analysis of the crank-triangular linkage-elbow mechanism}

\subsection{Crank-triangular linkage-elbow mechanism and its decomposition}

As shown in Figure 3, a CTLE has upper, middle and lower elbow levers with lengths $l_{1}, l_{2}$ and $l_{3}$ respectively, and the pivot $O$ at the upper end of the upper elbow lever is grounded. The lower elbow lever pivot $\mathrm{O}_{2}$ is fixed to the slide. $L_{1}$ and $L_{2}$ stand respectively for the lengths of the upper and lower sides of the triangular linkage, and the bottom side is the same as the middle elbow lever $l_{2}$. The center $O_{1}$ of crank $R$ is grounded, and the end of the crank is hinged to end $A$ of the triangular linkage. The input parameters of the CTLE include crank angle $\alpha$, rotational speed $\omega$ and torque $T$. The output parameters are slide stroke $S$, speed $V$ and pressure $P$. The solid lines show the slide's position at BDC, while the dotted lines show the slide position at TDC.

From the preliminary analysis of the CTLE, it can be seen that, in addition to the driving element (crank), the mechanism also consists of two Assur groups: one is the

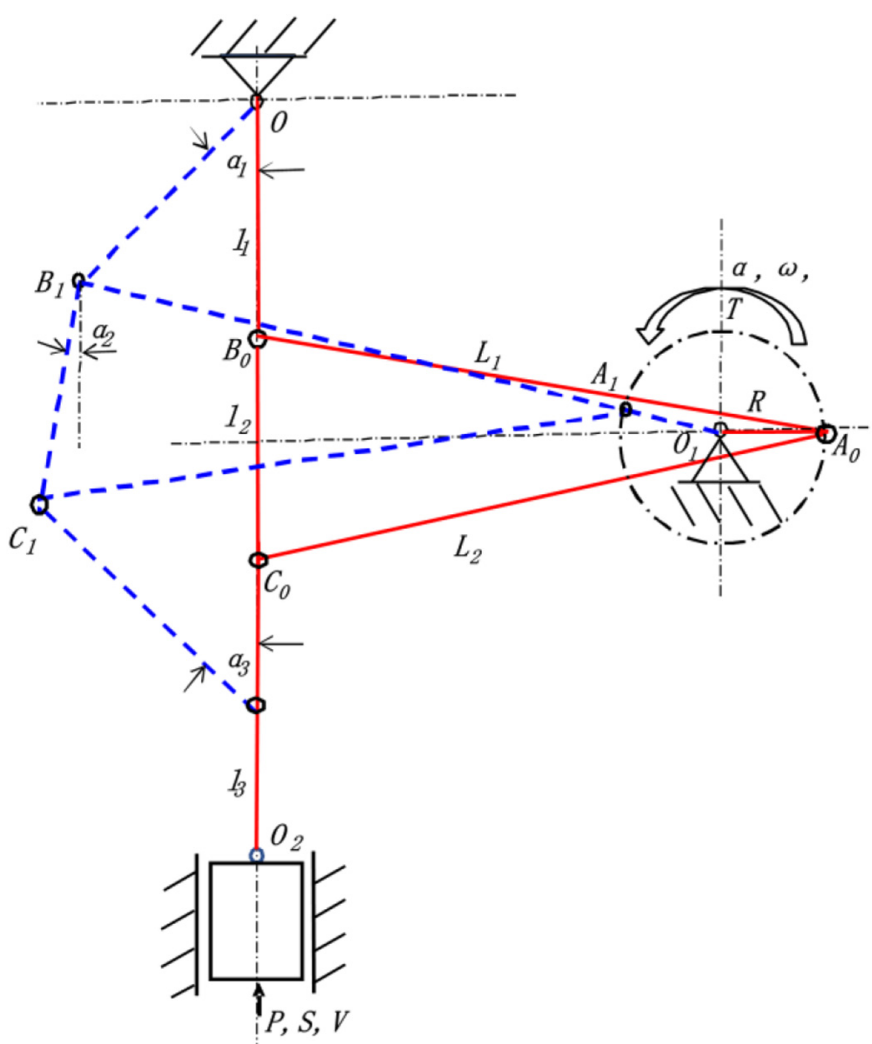

Fig. 3. Schematic diagram of crank-triangular linkage-elbow mechanism

rocker mechanism composed of the triangle link $A B C$ and the upper elbow bar $O B$; the other is the link-slide mechanism composed of the lower elbow bar $\mathrm{CO}_{2}$ and the slide. Usually, in the mechanical press, the combination form of the two groups is fixed, namely, at BDC four points $\left(\mathrm{O}, \mathrm{B}, \mathrm{C}\right.$ and $\left.\mathrm{O}_{2}\right)$ are on the same plumb line $O O_{2}$. To simplify the analysis, the whole mechanism is only divided into two subunits: Subunit 1, the driving element crank $R$ (Fig. 4b) and subunit 2, which is a combination of two Assur groups with 0 DOF: triangular connecting link-elbow and rod-slide (Fig. 4a).

\subsection{Analysis of subunit 1}

As shown in Figure 4b, the $X_{1} O_{1} Y_{1}$ coordinate system is established to describe the motion trajectory of point $A$, which is a circular motion with $O_{1}$ as its center and $R$ as its radius. The inputs of subunit 1 are crank angle $\alpha$, rotation 


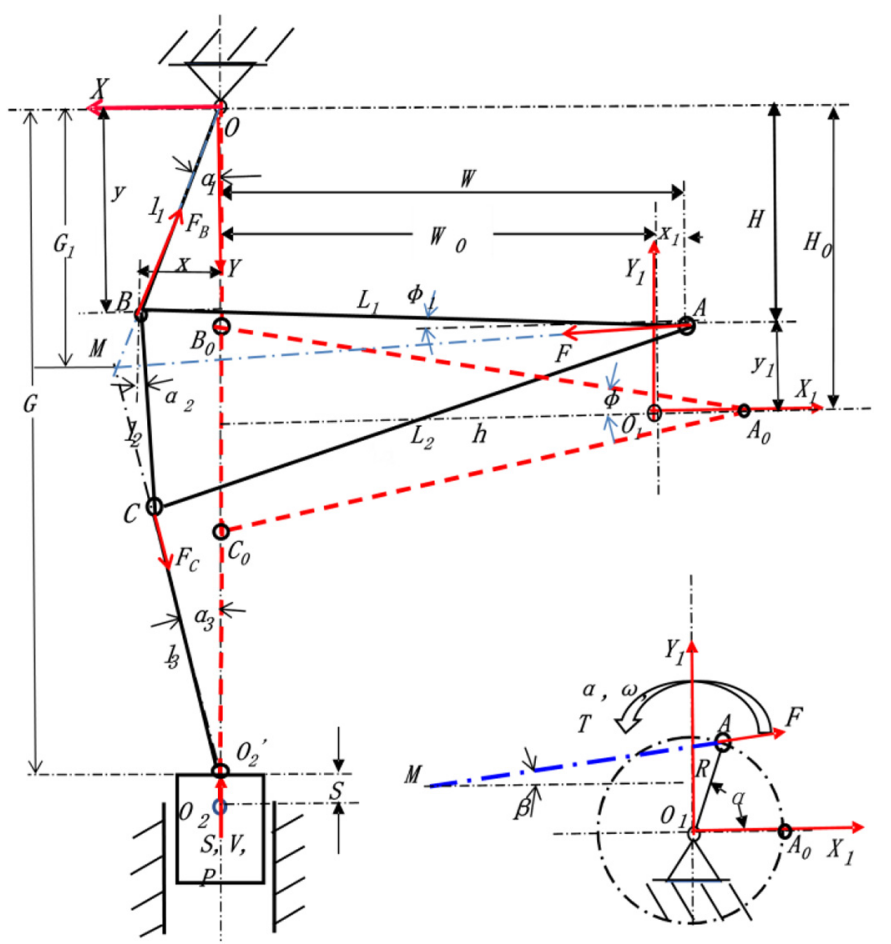

(a)

(b)
Fig. 4. Decomposition of crank-triangular linkage-elbow mechanism. (a) Triangular linkage-elbow. (b) Crank.

speed $\omega$ and torque $T$. The outputs are displacements $\left(x_{1}, y_{1}\right)$, speeds $\left(v_{1 x}, v_{1 y}\right)$ and force $F$ of point $A$. The direction of $F$ is determined by subunit 2, and the included angle between $F$ and horizontal line is $\beta$. After analysis, it is not difficult to obtain equations (4)-(6).

$$
\begin{gathered}
\left\{\begin{array}{l}
X_{1}=R \cos \alpha \\
Y_{1}=R \sin \alpha
\end{array}\right. \\
\left\{\begin{array}{l}
V_{1 X}=-R \omega \sin \alpha \\
V_{1 Y}=R \omega \cos \alpha
\end{array}\right. \\
\left\{\begin{array}{l}
F=\frac{T}{R \sin (\alpha-\beta)} \\
M A_{1}=\frac{F}{T}=\frac{1}{R \sin (\alpha-\beta)} .
\end{array}\right.
\end{gathered}
$$

Based on equations (4)-(6), it can be deduced that: (1) with $R$ or $(\alpha-\beta)$ decreasing, $M A_{1}$ will increase; (2) the position of the maximum mechanical advantage $M A_{1 \max }$ is located at $\alpha-\beta=k \pi, k=0,1,2, \ldots$, i.e., the crank and the action line of the output force coincide; (3) the maximum output displacement and velocity will decrease with $R$ reducing.

\subsection{Analysis on subunit 2}

\subsubsection{Kinetic analysis}

As shown in Figure 4a shows, the inputs of subunit 2 are the outputs of subunit 1 , and the outputs refer separately to slide stroke $S$, speed $V$ and pressure $P$. By setting the grounded pivot $O$ of the upper elbow lever as the origin, the $X O Y$ coordinate system is established. $A_{0} B_{0} C_{0}$ represents the initial position (BDC) of the triangle and $A B C$ an arbitrary position. $W_{0}$ and $H_{0}$ stand for the horizontal and vertical distances between crank center $O_{1}$ and grounded pivot $O$ on the elbow lever respectively; $W$ and $H$ are the horizontal and vertical distances between crank end $A$ and grounded pivot $O$ on the elbow lever respectively, while $\alpha_{1}$, $\alpha_{2}$ and $\alpha_{3}$ are the angles between the upper, middle and lower elbow levers and the plumb line $\mathrm{OO}_{2}$ respectively. The motion trajectory $(x, y)$ of point $B$ is determined by the rocker mechanism composed of $A B O$, which is a swing around pivot $O$. For kinematic analysis, equation (7) is established.

$$
\left\{\begin{array}{l}
x^{2}+y^{2}=l_{1}^{2} \\
(x+W)^{2}+(H-y)^{2}=L_{1}^{2}
\end{array}\right.
$$

By solving equation (7), the motion trajectory of point $B$ can be obtained and then the kinematic parameters such as displacement and velocity of slide can be deduced. As the final expressions are slightly complex, they are not listed here.

\subsubsection{Force analysis and simplification}

As shown in Figure 4a, the three forces acting on the vertices $A, B$ and $C$ stand for $F, F_{B}$ and $F_{C}$ respectively. If the friction and inertia are ignored, the three forces will converge at point $M$, which is the intersection of the extension line $l_{1}$ and $l_{3}$. $A M$ will be the acting line of $F$. Thus, a CTLE will become equivalent to a conventional crank-linkage-elbow mechanism (CLEM), as shown in Figure 5. The horizontal distance between point $M$ and line $\mathrm{OO}_{2}$ is $x$ '. The lengths of the upper and lower equivalent elbow levers are $l_{1}$ ' and $l_{3}$, while the linkage length is $L$. Unlike a real conventional CLEM, $l_{1}$,,$l_{3}$ ' and $L$ vary with the movement of the mechanism.

See Figure 4a, the equivalent CLEM is geometrically analyzed and the structural parameters are obtained by equations (8) and (9):

$$
G=l_{1} \cos \alpha_{1}+l_{2} \cos \alpha_{2}+l_{3} \cos \alpha_{3}
$$

$$
G_{1}=\frac{\tan \alpha_{3}}{\tan \alpha_{1}+\tan \alpha_{3}} G
$$

$$
\beta=\tan ^{-1} \frac{y_{1}-H_{0}+G_{1}}{W_{0}+x_{1}+G_{1} \tan \alpha_{1}} .
$$

Here $G_{1}$ is the vertical distance between point $M$ and ground pivot $O$, and $G$ is the vertical distance between $\mathrm{O}_{2}$, and $O$. 


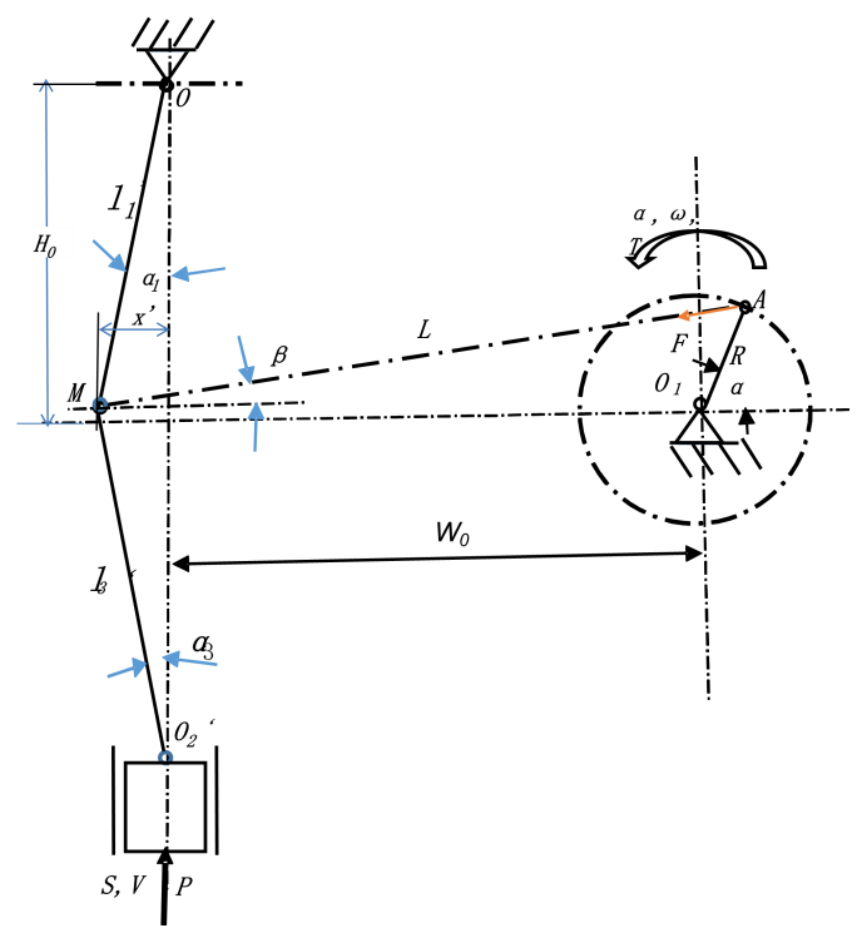

Fig. 5. Equivalent crank-linkage-elbow mechanism.

After analyzing the balance of three forces $F, F_{B}$ and $F_{C}$, the mechanical advantage of subunit 2 and the slide stroke can be obtained by equations (11) and (12).

$$
\begin{aligned}
& F_{C}=\frac{\cos \left(\alpha_{1}+\beta\right)}{\sin \left(\alpha_{1}+\alpha_{3}\right)} F \\
& P=F_{C} \cos \alpha_{3}=\frac{\cos \left(\alpha_{1}+\beta\right) \cos \alpha_{3}}{\sin \left(\alpha_{1}+\alpha_{3}\right)} F \\
& M A_{2}=\frac{P}{F}=\frac{\cos \left(\alpha_{1}+\beta\right) \cos \alpha_{3}}{\sin \left(\alpha_{1}+\alpha_{3}\right)} \\
& S=l_{1}^{\prime}\left(1-\cos \alpha_{1}\right)+l_{3}^{\prime}\left(1-\cos \alpha_{3}\right) .
\end{aligned}
$$

\subsubsection{Performance features of equivalent linkage-elbow mechanism}

(1) From Figure 4a and equation (11), it can be seen that when $\alpha_{1}=\alpha_{2}=0, \alpha_{3}$ must be 0 and the slide is at BDC. In other words, when $\alpha_{1}=\alpha_{2}=\alpha_{3}=0$, subunit 2 will get the maximum mechanical advantage, i.e., $M A_{2}=\infty$.

(2) By substituting $\cos \alpha_{1}=\frac{\sqrt{l_{1}^{\prime 2}-x^{\prime 2}}}{l_{1}^{\prime}}, \cos \alpha_{3}=\frac{\sqrt{l_{3}^{\prime 2}-x^{\prime 2}}}{l_{3}^{\prime}}$, $\sin \alpha_{1}=\frac{x^{\prime}}{l_{1}^{\prime}}$ and $\sin \alpha_{3}=\frac{x^{\prime}}{l_{3}^{\prime}}$ into equation (11) it can be known that $\frac{\partial M A_{2}}{\partial l_{1}^{\prime}} \geqq 0, \frac{\partial M A_{2}}{\partial l_{3}^{\prime}} \geqq 0$, which means that the mechanical advantage $M A_{2}$ is a monotonically increasing function of lever length $l_{1}$ 'and $l_{3}$ '. With the increase of lever length $l_{1}$ ' or $l_{3}$, $M A_{2}$ increases. In practice, due to the structural constraints, both $l_{1}$ 'and $l_{3}$ 'should be limited to a certain range.

(3) By using a similar way to analyze equation (12), it can be proved that slide stroke $S$ is a monotonically decreasing function of $l_{1}$ ' and $l_{3}$ '. $S$ and $S$ max will decrease with the increasing of $l_{1}$ ' or $l_{3}$ '.

(4) When the slide approaches BDC, $\beta$ is small, $\beta \approx 0$. It can be proved from equation (11) that when $l_{1}{ }^{\prime}=l_{2}{ }^{\prime}\left(\alpha_{1}=\right.$ $\left.\alpha_{2}=\alpha\right), M A_{2}$ will get the maximum, $M A_{2 \max }=\frac{\cos ^{2} \alpha}{\sin 2 \alpha}$.

(5) Usually, since $0<\alpha 1<\pi / 2$, it can be seen from equation (11) that higher $M A_{2}$ will be achieved when $\beta<0$. But it will be the contrary if the rotation direction is reversed.

To get the total mechanical advantage, equation (13) is obtained by combining equation (2), equation (6) and equation (11).

$$
M A=M A_{1} \bullet M A_{2}=\frac{\cos \left(\alpha_{1}+\beta\right) \cos \alpha_{1}}{R \sin (\alpha-\beta) \sin \left(\alpha_{1}+\alpha_{3}\right)} .
$$

\section{Peak stagger design for crank-triangle linkage-elbow mechanism}

\subsection{Offset analysis of crank-triangular linkage-elbow mechanism}

In addition to the structural parameters, the form of subunit combination also has a great influence on the performance. The combination of the two subunits for a CTLE might have three arrangements (Fig. 6), where the height of triangle $A B C$ is $h$. When the slide is located at $\mathrm{BDC}$, the three elbow levers $l_{1}, l_{2}$ and $l_{3}$ will be collinear. The concept of the offset of the slide-crank mechanism [33] is extended to the CTLE. When the slide locates at BDC, if pivot $A$ locates on the crank horizontal center line, such a CTLE is defined as the in-line CTLE (Fig. 6a). On the contrary, if pivot $A$ is out of the center line, the mechanism is called offset CTLE, and the vertical distance $e$ between pivot $A$ and crank center $O_{1}$ is called the offset distance. If pivot $A$ is located above the center line, it is called the positive offset (Fig. 6c), $e>0$. Conversely, it is called the negative offset (Fig. 6d), $e<0$. In the common mechanical press with CTLE, most of the mechanisms are in-line.

As mentioned above, the CTLE can be considered as a combination of the two subunits, with the mechanical advantage $M A_{1}$ and $M A_{2}$ respectively. The mechanical advantage of the whole mechanism will be $M A=M A_{1}$. $M A_{2}$. Figure 7 is a schematic diagram of the relationship among $M A_{1}, M A_{2}$ and $M A$. As for the in-line mechanism, a higher $M A$ can be obtained at BDC, since both $M A_{1}$ and $M A_{2}$ reach their peaks at $\mathrm{BDC}$, but $M A$ will drop rapidly when the slide deviates from $\mathrm{BDC}$, since both $M A_{1}$ and $M A_{2}$ drop with a great rate. So, $M A$ will not be high enough at $S_{n}$ (Fig. 7a). In regard to the positive offset mechanism, $M A_{1}$ will reach its peak at the slide stroke $S_{1}$ before $M A_{2}$ does. If $S_{1}$ and $S_{n}$ are approximately coincident, there will be a relatively high and steady $M A$ in the entire $S_{n}$, as displayed in Figure $7 \mathrm{~b}$. As for the negative offset mechanism, it is not helpful for increasing $M A$ in $S_{n}$, since $M A_{1}$ reaches its peak when the slide is already in the return stroke. 


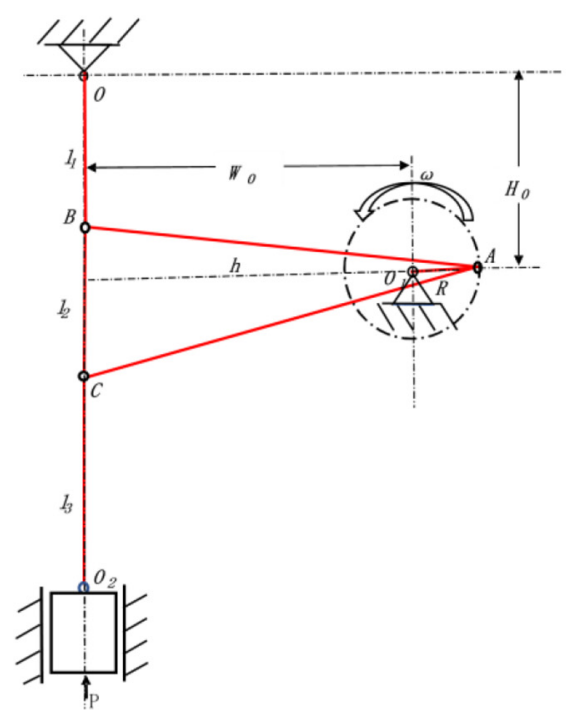

(a)

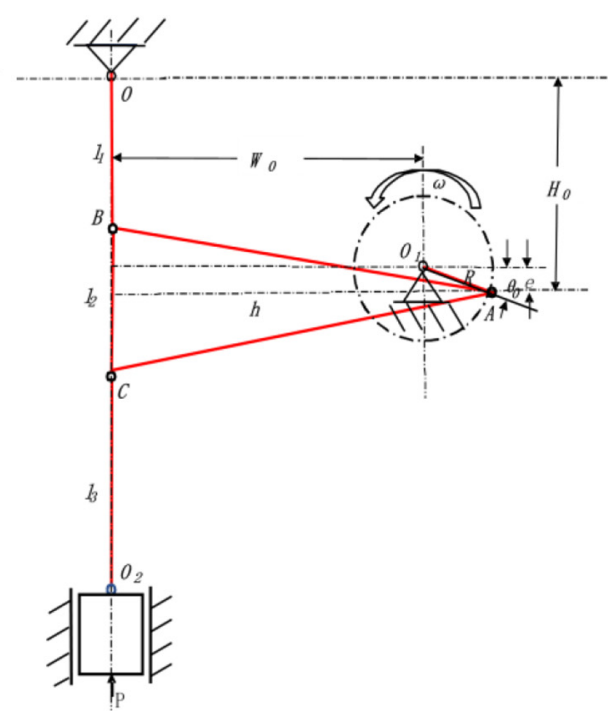

(b)

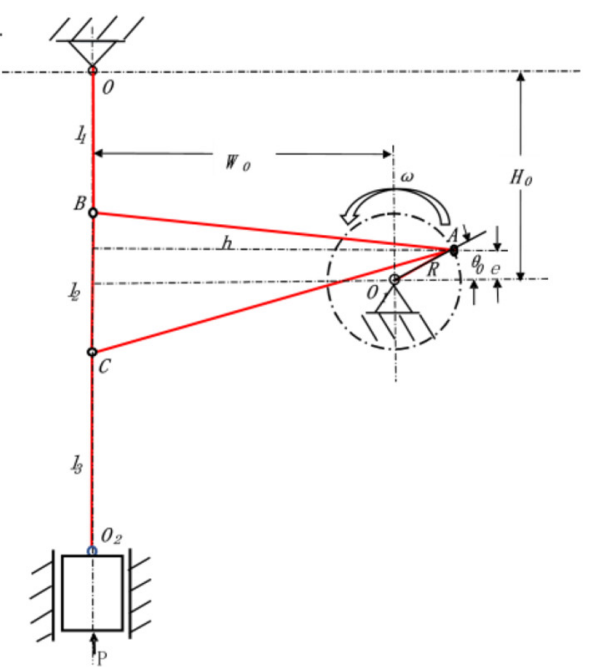

(c)

Fig. 6. Structural forms of crank-triangular link-elbow mechanism. (a) In-line. (b) Negative offset. (c) Positive offset.

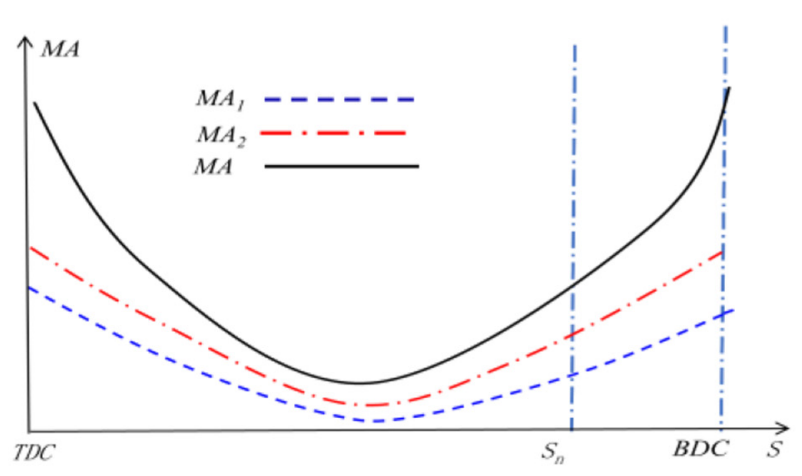

(a)

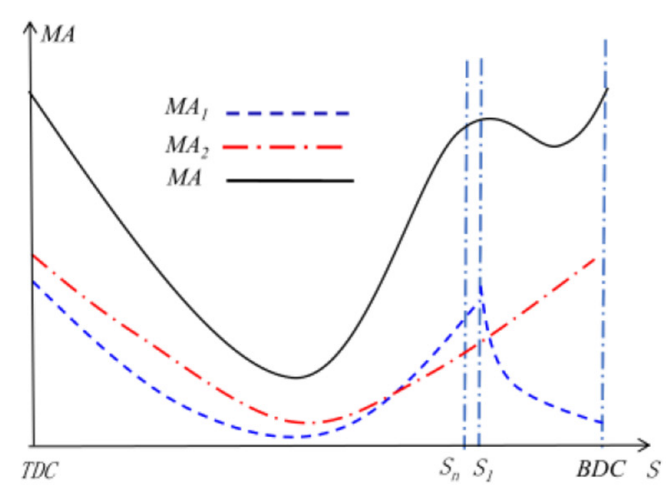

(b)

Fig. 7. Synthesis of mechanical advantages. (a) In-line. (b) Positive offset.

\subsection{Peak stagger design for crank-triangle linkage-elbow mechanism}

The mechanism is designed with peak stagger (positive offset) to ensure that $M A_{1 \max }$ occurs before $M A_{2 \max }$. As laid out in Figure $6 \mathrm{c}$, the mechanism offset angle is $\theta_{0}$, then equation (14) is obtained.

$$
\theta_{0}=\sin ^{-1} \frac{e}{R}
$$

where $e$ is the offset distance and $R$ is the crank radius. $\Delta \alpha$ is the included angle between the two peaks, which is called the stagger angle, expressed by equation (15).

$$
\Delta \alpha=\theta_{0}-\beta_{0}
$$

where $\beta_{0}$ is the angle between the crank and the horizontal line when subunit 1 reaches its peak value. In the preliminary design, the stagger angle $\Delta \alpha$ should be slightly less than the nominal pressure angle $\alpha_{n}$, placing the two peaks within working stroke $S_{n}$. Thus, a high and steady mechanical advantage in the entire working stroke can be achieved.

According to equation (3), the slide has not only a large and steady mechanical advantage within $S_{n}$, but also a low and steady speed, meeting the requirements of the forming process.

\section{Design examples}

In order to further verify the analysis in Section 4, three CTLE models are designed for comparison (shown in Tab. 2), and scheme $\mathrm{a}, \mathrm{b}$ and $\mathrm{c}$ correspond to the mechanisms shown in Figure $6 \mathrm{a}, 6 \mathrm{~b}$ and $6 \mathrm{c}$ respectively. Their main structural parameters are determined by 
Table 2. Structural dimensions of three types of crank-triangular linkage-elbow mechanism $/ \mathrm{mm}$.

\begin{tabular}{llll}
\hline Scheme & a. in-line & b. neg. offset & c. pos. offset \\
\hline$l_{1}$ & 380 & 380 & 380 \\
$l_{2}$ & 300 & 300 & 300 \\
$l_{3}$ & 420 & 420 & 420 \\
$W_{0}$ & 880 & 880 & 880 \\
$H_{0}$ & 530 & 460 & 610 \\
$R$ & 130 & 130 & 150 \\
$e$ & 0 & -70 & 80 \\
\hline
\end{tabular}

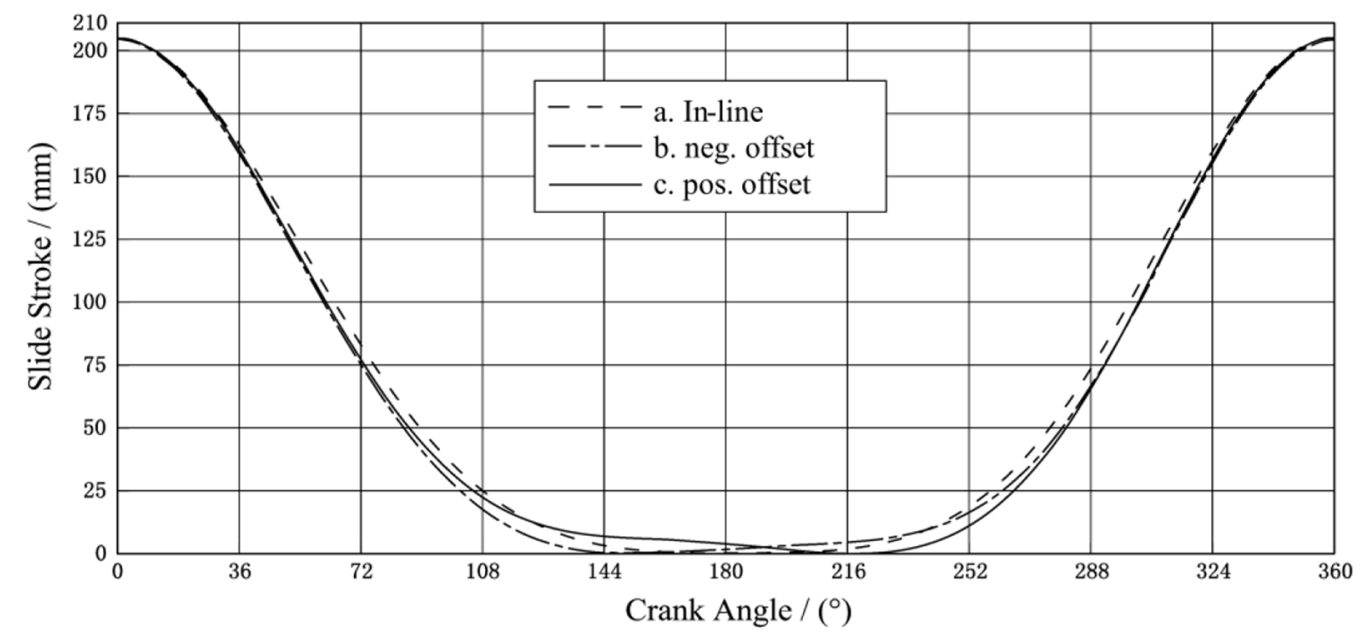

Fig. 8. Slide stroke curves of three mechanisms.

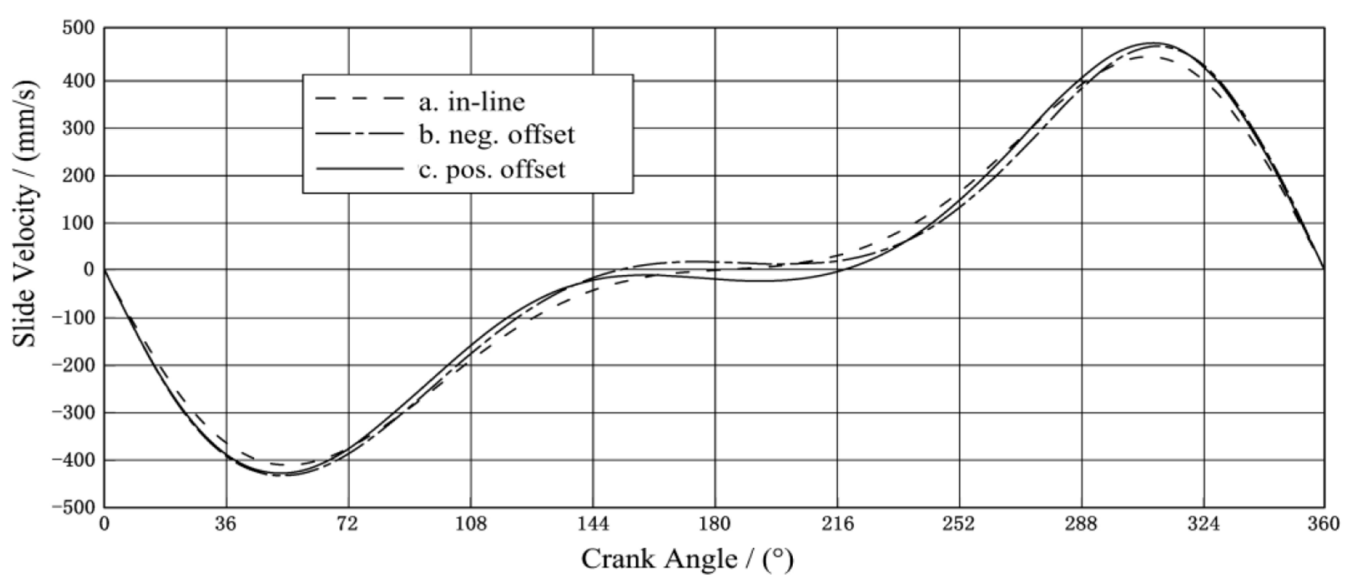

Fig. 9. Slide velocity curves of three mechanisms.

referring to the structure of an existing servo mechanical press with the same tonnage [11]. The main parameters of the three schemes are approximately the same, but $H_{0}$ is different to realize the different offset. The maximum slide strokes $S_{\max }$ of the three schemes are kept at about $200 \mathrm{~mm}$ by adjusting the crank radius $R$. The working parameters are: $P_{n}=4000 \mathrm{kN}, S_{n}=6 \mathrm{~mm}$, the working frequency is 30 SPM (Stroke Per Minute).
By software VI-Automotives ${ }^{\circledR}$ [34], the kinematics and forces of the three mechanisms are numerically simulated, ignoring the friction and mass. Figures 8-10 show the slide stroke, speed and mechanical advantage of the three mechanisms, and Figure 11 shows the required input torque for output $P_{n}$ in $S_{n}$. Table 3 shows the comparison of working performance of the three schemes. 


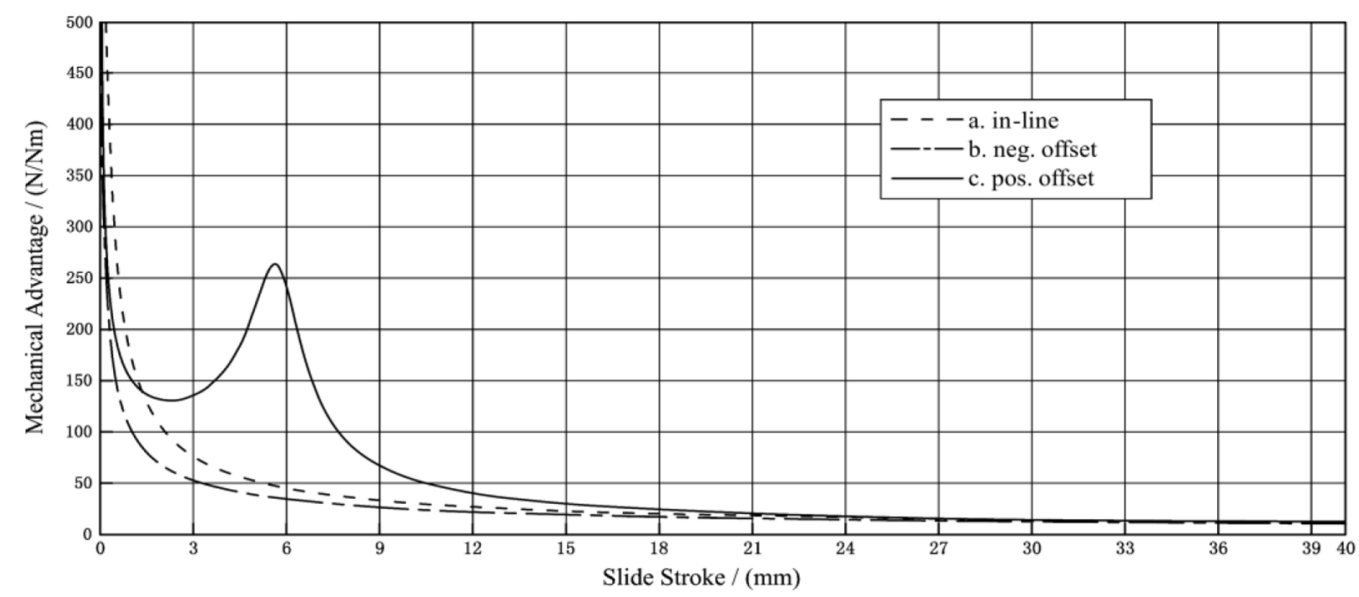

Fig. 10. Mechanical advantage-stroke curves of three mechanisms.

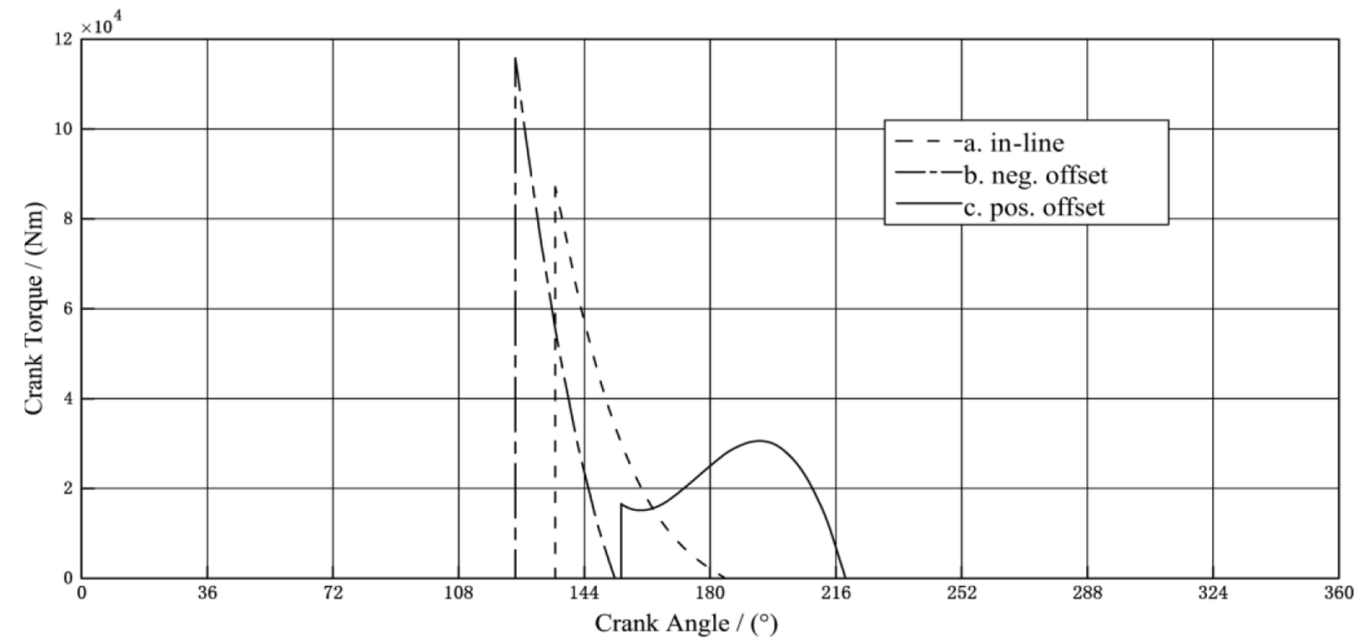

Fig. 11. Input torque required for nominal pressure output.

Table 3. Performance comparison of three schemes.

\begin{tabular}{lllll}
\hline Scheme & $\mathrm{a}$ & $\mathrm{b}$ & $\mathrm{c}$ & Note \\
\hline$S_{\text {max }, m m}$ & 203.4 & 203.3 & 204.1 & Maximum stroke \\
$\bar{V}_{, m m / s}$ & -22.33 & -38.02 & -16.87 & Average velocity in $S_{n}$ \\
$S D V_{, m m / s}$ & 19.50 & 26.51 & 5.58 & Velocity standard deviation in $S_{n}$ \\
$M A_{n}, N / N_{m}$ & 45.7 & 34.6 & 241.7 & $M A$ at $S_{n}$ \\
$M A_{\text {min }}, N / N_{m}$ & 19.5 & 34.6 & 129.7 & The minimum $M A$ in $S_{n}$ \\
$T_{n}, N_{m}$ & 87,075 & 115,730 & 30,547 & Required torque input in $S_{n}$ \\
\hline
\end{tabular}

As can be seen from Table 3, the positive mechanism (Scheme c) has the best performances. In comparison with the in-line mechanism (Scheme a), its $M A_{n}\left(M A\right.$ at $\left.S_{n}\right)$ increases from $45.7 \mathrm{~N} / \mathrm{Nm}$ to $241.7 \mathrm{~N} / \mathrm{Nm}$, an increase of $428 \%$, while $\bar{V}$ (average slide speed in $S_{n}$ ) decreases from $22.3 \mathrm{~mm} / \mathrm{s}$ to $16.9 \mathrm{~mm} / \mathrm{s}$, a decrease of $24.2 \%$, and $S D V$ (the standard deviation of the slide speed in $S_{n}$ ) decreases from $19.5 \mathrm{~mm} / \mathrm{s}$ to $5.6 \mathrm{~mm} / \mathrm{s}$, a decrease of $85.6 \%$.
Contrarily, for the negative offset (scheme b), $M A_{n}$ decreases from $45.7 \mathrm{~N} / \mathrm{Nm}$ to $34.6 \mathrm{~N} / \mathrm{Nm}$, a decrease of $24.3 \%$, while $\bar{V}$ increases from $22.3 \mathrm{~mm} / \mathrm{s}$ to $38.0 \mathrm{~mm} / \mathrm{s}$, an increase of $70.4 \%$, and $S D V$ increases from $19.5 \mathrm{~mm} / \mathrm{s}$ to $26.5 \mathrm{~mm} / \mathrm{s}$, an increase of $35.9 \%$.

Figure 10 shows that $M A$ of Scheme c fluctuates with two peaks in $S_{n}(0-6 \mathrm{~mm})$. The first peak is at about $5.5 \mathrm{~mm}$ of the slide stroke and the second at BDC. Even in such a 
situation, the minimum value of $M A$ (wave trough) in $S_{n}$ still reaches $129.7 \mathrm{~N} / \mathrm{Nm}$, which is $184 \%$ higher than that of the in-line arrangement.

From Figure 11 it can be seen that in order to get the pressure output of $4000 \mathrm{kN}$ in $S_{n}$, the maximum input torque for Scheme a is $87,075 \mathrm{Nm}$. However, it is only $30,574 \mathrm{Nm}$ for Scheme c, with a decrease of $65 \%$.

With the peak stagger design, the CTLE can get high and steady mechanical advantage, low and uniform slide velocity in the entire working stroke, which is of great significance for servo mechanical presses to reduce the motor capacity and improve their working performance.

It should be pointed out that Scheme $\mathrm{c}$ is only a preliminary design, and the performance can be further improved by adjusting the structural parameters through subsequent computer optimization design.

\section{Conclusion}

A new design method of multi-linkage mechanism based on decomposition analysis is proposed, which provides a basis for building a preliminary model for subsequent optimization design, so as to improve the design efficiency.

Different from the traditional decomposition method, the main purpose of the new method is to obtain the influence of both structural parameters and interconnecting form of each sub-unit on the performance, which can be used as a guide for the preliminary design. The submechanism can be either an Assur group or a combination of several Assur groups with fixed connection modes.

By the decomposition method, the crank-triangular linkage-elbow mechanism commonly used in servo presses is analyzed and the influence of structural parameters on the working performance of the mechanism is obtained.

Based on the decomposition analysis, the peak stagger design method for crank-triangular linkage-elbow mechanism is established. By offset design, the mechanical advantage peaks of the two sub-mechanisms are staggered: $M A_{1 \text { max }}$ is located near $S_{n}$ while $M A_{2 \max }$ just at BDC. The stagger angle $\Delta \alpha$ is slightly less than the nominal pressure angle $\alpha_{n}$. By stagger peak design, the working property can be improved, while the input torque will be decreased, reducing the press cost.

The design example verified the feasibility of the stagger peak design method: compared with the in-line design, in the entire working stroke the minimum mechanical advantage increases by $184 \%$, the average slide speed decreases by $24.2 \%$, the standard deviation of the speed decreases by $85.6 \%$, while the input torque decreases by $65 \%$.

\section{Availability of data and materials}

The datasets used and analyzed during the current study are available from the corresponding author on reasonable request.

\section{Competing interests}

The authors declare that they have no competing interests.

This research has been financed by the National Natural Science Foundation of China (51175088) and Guangdong Province Science and Technology Research Project (2009Z019), and the authors would like to express their heartfelt thanks for these.

\section{Author contribution statement}

YS put forward the main academic ideas and was in charge of the theoretical analysis and the article writing. JH was in charge of the digital simulation. LW took part of the theoretical analysis. YC took part of digital simulation.

\section{References}

[1] Y. Sun, J. Hu, Y. Cheng, Characteristics analysis of working mechanisms for servo mechanical presses, Appl. Mech. Mater. 220-223, 762-767 (2012)

[2] Schuler, Metal forming handbook, Springer-vertag, Berlin Heidelber, 1998

[3] K. Kawamotoa, H. Andob, K. Yamamichia, Application of servo presses to metal forming processes, Proc. Manufactur. 15, 31-38 (2018)

[4] K. Osakada, K. Mori, T. Altan, Mechanical servo press technology for metal forming, CIRP Ann. Manufactur. Technol. 60, 651-672 (2011)

[5] R. Halicioglu, L.C. Dulger, A.T. Bozdana, Mechanisms, classifications, and applications of servo presses: a review with comparisons, Proc. Inst. Mech. Eng. B 230, 1177-1194 (2016)

[6] C.-F. Meng, C. Zhang, Y. Lu, Z. Shen, Optimal design and control of a novel press with an extra motor, Mech. Mach. Theory 39, 811-818 (2004)

[7] S.G. Bosga, M. Segura, Design and tests of a hybrid servo drive system for a1000 $\mathrm{T}$ mechanical press, in Power Electronics Specialists Conference, PESC 2008. IEEE 5-19, 424-429 (2008)

[8] Y. Bai, F. Gao, W. Guo, Design of mechanical presses driven by multi-servo motor, J. Mech. Sci. Technol. 25, 2323-2334 (2011)

[9] J. Liang, S. Zhao, Y. Zhao et al., Hybrid-loop servo control system of double toggle mechanical press for flexible forming process based on sliding mode control and neural network techniques, Proc. IMechE Part I 230, 35-45 (2016)

[10] A. Tuleshov, R. Halicioglu, A. Shadymanova et al., Kinematic synthesis method and eccentricity effects of a Stephenson mechanism, Mech. Sci. 12, 1-8 (2021)

[11] J. Hu, Y. Sun, Y. Cheng, High mechanical advantage design of six-bar Stephenson mechanism for servo mechanical presses, Adv. Mech. Eng. 8, 1-12 (2016)

[12] J. Mo, Z. Zhang, Y. Lv et al., The simulation and optimization of triangle toggle rod transmission mechanism for servo press (in Chinese), China Metal Forming Equip. Manufactur. Technol. 2011, 21-25 (2011) 
[13] V. García-Marina, I. Fernández de Bustos, G. Urkullu et al., Optimum dimensional synthesis of planar mechanisms with geometric constraints, Meccanica 55, 2135-2158 (2020)

[14] R. Halicioglu, L. Canan Dulger, A. Tolga Bozdana, Modeling, design, and implementation of a servo press for metal-forming application, Int. J. Adv. Manufactur. Technol. 91, 2689-2700 (2017)

[15] A. Jomartov, A. Tuleshov, N. Jamalov et al., Dynamic model of servo mechanical press, in: ROMANSY 2020, CISM 601, edited by G. Venture et al., 2021, pp. 170-178

[16] A.A. Jomartov, A.K. Tuleshov, M.Zh. Kuatova, Simulation of the crank press dynamics by SimulationX software, J. Math. Mech. Comput. Sci. 2, 22-33 (2019)

[17] Y. Zhou, F. Xie, X. Liu, Type synthesis and optimization of main driving mechanism for servo-punch press (in Chinese), J. Mech. Eng. 51, 1-7 (2015)

[18] A.K. Tuleshov, A.A. Jomartov, S. Ibrayev et al., Optimal synthesis of planar linkages, NEWS of the Academy of Sciences of the Republic of Kazakhstan 1, 172-180 (2020)

[19] M.K. Sonpimple, P.M. Bapat, J.P. Modak et al., A novel hybrid simulated annealing-direct search algorithm for optimum synthesis of function generation problem, J. Theor. Appl. Inf. Technol. 11, 16-24 (2010)

[20] A. Höber, B.-A. Behrens, R. Krimm, Development of a design tool for Servo-powertrains in forming presses, Proceedings of the 9th Congress of the German Academic Association for Production Technology (WGP): Production at the leading edge of technology, Hamburg, Germany, September 30th - October 2nd, 2019, pp. 93-101

[21] P.L. Tso, K.C. Liang, A nine-bar linkage for mechanical forming presses, Int. J. Mach. Tools Manufact. 42, 139-145 (2002)

[22] K.C. Ham, D.H. Jang, Kinematical analysis on the several linkage drives for mechanical presses, J. Mech. Sci. Technol. 23, 512-524 (2009)
[23] W.M. Hwang, Y.C. Hwang, S.T. Chiou, A drag-link drive of mechanical presses for precision drawing, Int. J. Mach. Tools Manufact. 35, 1425-1433 (1995)

[24] W.H. Hsieh, C.H. Tsai, Optimum design of a novel press system with Stephenson-I mechanism, Comput. Math. Appl. 64, 897-907 (2012)

[25] S. Yossifon, R. Shivpuri, Analysis and comparison of selected rotary linkage drives for mechanical presses, Int. J. Mach. Tools Manufact. 33, 175-192 (1993)

[26] S. Yossifon, R. Shivpuri, Optimization of a double knuckle linkage drive with constant mechanical advantage for mechanical presses, Int. J. Mach. Tools Manufact. 33, 193-208 (1993)

[27] W.Z. Guo, F. Gao, Design of a servo mechanical press with redundant actuation, Chin. J. Mech. Eng. 22, 574-579 (2009)

[28] R. Du, W.Z. Guo, The design of a new metal forming press with controllable mechanism, J. Mech. Des. 125, 582-592 (2003)

[29] Z. Wang, J. Mo, Performance research of triangular transmission system of servo press, China Metal Form. Equip. Manufactur. Technol. 50, 13-16 (2015)

[30] H. Sun, Z. Chen, Principle of Machinery, Chinese Higher Education Press, Beijing, 1996, (in Chinese)

[31] M.R. Hansen, A general method for analysis of planar mechanisms using a modular approach, Mech. Mach. Theory 31, 1155-1166 (1996)

[32] S. Li, H. Wang, Q. Yang, Constraint force analysis of metamorphic joints based on the augmented Assur groups, Chin. J. Mech. Eng. (English Edition) 28, 747-755 (2015)

[33] H.D. Eckhardt, Kinematic Design of Machines and Mechanisms, McGraw-Hill Education Co. \& China Machine Press, Beijing, China, 2003

[34] Real-time and multibody system modeling software: VI-Automotives $[\mathrm{CP} / \mathrm{CD}]$ VI-grade $\mathrm{p}$ roduct by VI-grade Company (2016)

Cite this article as: Y. Sun, J. Hu, L. Wei, Y. Chen, Decomposition analysis and peak stagger design for Crank-triangular linkageelbow mechanism of mechanical servo presses, Mechanics \& Industry 22, 43 (2021) 